Review

\title{
High-speed Orbital Angular Momentum Multiplexed Free-space Optical communication systems
}

\author{
Zhen $\mathrm{Qu}$ * and Ivan B. Djordjevic \\ University of Arizona, Department of Electrical and Computer Engineering, 1230 E. Speedway Blvd., Tucson, \\ AZ 85721, USA \\ * Correspondence: zhenqu@email.arizona.edu; Tel.: +1-520-442-7197
}

\begin{abstract}
We review recent progress in high-speed orbital angular momentum (OAM) multiplexed free-space optical communication systems. The outdoor atmospheric turbulence is emulated by an indoor turbulence emulator, which is based on split-step beam propagation method. Adaptive optics, channel coding, Huffman coding combined with LDPC coding, and spatial offset are used for turbulence mitigation; while OAM multiplexing and wavelength-division multiplexing (WDM) are applied to boost aggregate capacity.
\end{abstract}

Keywords: Orbital angular momentum; free-space optical communications; turbulence mitigation

\section{Introduction}

Optical communication systems are usually deployed over fiber-optic links [1-4] and free-space optical links [5-7]. Recent advances in photonics integrated circuits (PIC) have been greatly pushing forward the worldwide application of optical communications [8-10]. Although have enabled a capacity-approaching communication [11-13], fiber-optics links may be too fragile or costly to be deployed in some environments, e.g., seismic belts. As a result, FSO links may be more favorable because of the easy and fast communication link reconstruction. Although the channel loss of FSO links is not stable, and typically higher than that of a fiber-optic link, FSO communication systems provide free-scalable channels for spatial mode division (SDM) multiplexing, e.g., orbital angular momentum (OAM) multiplexing [14-17]. On the other hand, the multiplexed modes can't preserve the orthogonality in the presence of atmospheric turbulence, leading to inter-mode crosstalk [18,19].

To mitigate the crosstalk, adaptive optics (AO) [20,21], multi-input multi-output (MIMO) signal processing [22,23], and channel coding [24,25] are usually implemented to guarantee a reliable communication system. The AO system has been widely used in astronomy to reduce the wavefront distortion, while the costly wavefront sensor (WFS) and deformable mirror (DM) restrict its application in commercial FSO communication systems. MIMO equalization is a classical scheme to mitigate mode crosstalk, and it can work well in weak atmospheric turbulence. In case of middle and strong turbulence, mode crosstalk will not be confined to adjacent channels. Therefore, more detectors have to be employed to recover the signals in each channel, followed by more complex parallel channel equalization. Channel coding can be regarded as the last resort to realize an errorfree communication, but channel coding-only method hardly ensure a robust and error-free FSO communication, in particular in the presence of strong turbulence.

In this review paper, we discuss the high-speed OAM multiplexed FSO communication systems, enabled by AO, LDPC coding, spatial offset, and joint Huffman and LDPC coding. We first experimentally study an AO assisted, LDPC coded, OAM-based quadrature phase shift keying (QPSK) FSO transmission system. Four OAM modes each carrying 15.6 GBaud QPSK signals are transmitted on five $50 \mathrm{GHz}$-grid WDM channels to realize $500 \mathrm{~Gb} / \mathrm{s}$ FSO transmission system. The mode crosstalk is first mitigated by the wavefront sensorless AO system. Then additional system performance improvements are obtained by using low-density parity-check (LDPC) $(16935,13550)$ code. The coding gains of $3.9,4.1,5.2$, and $5 \mathrm{~dB}$ can be obtained at $\mathrm{BER}=2 \times 10^{-2}$ for OAM states of 2 , $-2,6$, and -6 , respectively. 
After that we present a two-stage crosstalk mitigation method in an OAM-based FSO communication system. The mode crosstalk is first mitigated in optical domain by spatial offset, and then relieved in digital domain by coded modulation. The 5-quadrature amplitude modulation (5QAM) and 9-QAM are used here based on Huffman coding and nonbinary LDPC coding [26-28]. Compared to the traditional systems, where the OAM beams are central aligned, and data are modulated with rectangular M-QAM formats, e.g., QPSK and 8-QAM, the proposed two-stage crosstalk mitigation method can largely improve the system performance.

\section{Adaptive optics enabled free-space optical communication}

\subsection{Experimental setup}

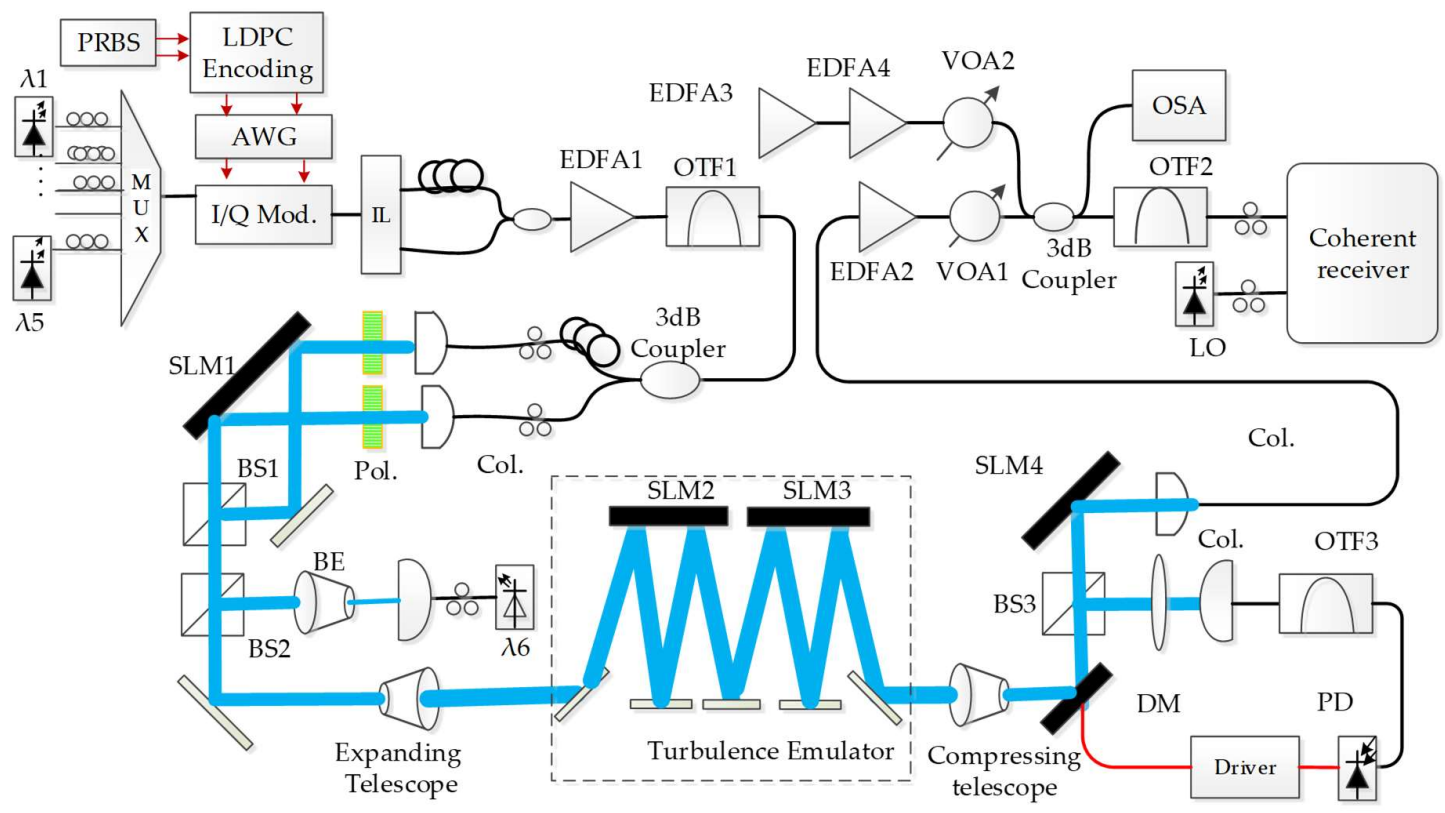

Figure 1. Experimental setup for AO enabled FSO communication system.

The experimental setup is shown in Figure 1. At the transmitter side, the continuous wave (CW) lights on a 50-GHz grid (193.50-193.30THz) are generated from five integrable tunable laser assembly (ITLA) lasers with a linewidth less than $10 \mathrm{kHz}$. The five channels are multiplexed and sent to an I/Q modulator to generate 15.6 GBaud wavelength-division multiplexing (WDM) QPSK signal. The PRBS data sequence is encoded by a binary quasi-cyclic LDPC code, i.e., LDPC $(16935,13550)$ code of girth8 and column-weight-3. Therefore, the QPSK signal with net bit rate of $25 \mathrm{~Gb} / \mathrm{s}$ is generated. A 50/100$\mathrm{GHz}$ optical interleaver (IL) is employed to separate the odd and even subchannels. Odd and even channels are then decorrelated by several hundreds of symbols via additional optical fiber and combined using a 3-dB coupler. The resulting WDM QPSK signals are amplified by an Erbium-doped fiber amplifier (EDFA), and filtered with an optical tunable filter (OTF). The filtered signal is split by a 3-dB optical coupler, followed by an optical tunable delay line applied in one path to decorrelate the data sequences. Each beam is collimated and converted to OAM beams by a reflective phase-only spatial light modulator (SLM). Note that the SLMs used offer $1920 \times 1080$ pixels' resolution, $15.36 \mathrm{~mm} \times 8.64 \mathrm{~mm}$ active area, and $60 \mathrm{~Hz}$ image frame rate.

The left and right halves of the SLM1 screen are loaded with different phase patterns to convert the incoming independent Gaussian beams to OAM beams (OAM modes with OAM state indices \pm 2 , \pm 6 ). A blazed "fork" phase pattern is used to separate the desirable OAM beams from the Gaussian beam. The generated OAM beams are then combined together by a beam splitter (BS). The other Gaussian beam with wavelength of $1548.9 \mathrm{~nm}$ acts as the probe beam for AO correction. This probe 
beam is expanded using a variable beam expander to become as wide as the widest OAM beam used in experiment, and then combined with the OAM beams.

After passing through the telescope system, the multiplexed beams are then reflected four times on the left and right halves of SLM screens over a total distance of two meters. The time-varying atmospheric turbulence is emulated by changing the random phase patterns at $50 \mathrm{~Hz}$ rate [21]. This turbulence model is built on the Rytov variance [29] of $\sigma_{R}^{2}=2$.

The OAM beams at the output of the turbulence emulator are collected by a 28-mm receiver aperture and pass through the compressing telescope. The beams are then imaged onto the DM for turbulence compensation. This wavefront sensorless AO system uses the aberrated probe beam as a feedback. Part of the beams will be separated by a BS and coupled to SMF. An OTF centered on 1548.9 $\mathrm{nm}$ is used to filter out the probe beam and the remaining signal is then detected by the PD. Finally, the captured photocurrent will be digitalized by a driver with sampling rate of $1 \mathrm{MSa} / \mathrm{s}$ to modify the DM based on stochastic parallel gradient descent (SPGD) algorithm.

After the wavefront correction, the multiplexed beams are sent to the fourth SLM loaded with a specified blazed "fork" phase pattern to demultiplex and detect (convert) one of the multiplexed OAM modes back to Gaussian beam, which is then coupled into the SMF.

At the receiver side, the received signal is amplified by an EDFA to compensate the loss and its power is adjusted by a VOA. The ASE noise-loading stage is constructed by two EDFAs cascaded with a VOA. The ASE noise and the signal are combined with a 3-dB coupler, followed by an OTF centered on $1550.12 \mathrm{~nm}$ to select the channel under test. Another laser with linewidth less than $10 \mathrm{kHz}$ serves as a LO. Two polarization controllers (PCs) are used to align the polarizations of the signal light and LO before being mixed in coherent receiver. The coherent receiver includes an optical $90^{\circ}$ hybrid, two $23 \mathrm{GHz}$ single-end PDs, and real-time oscilloscope with $100 \mathrm{GSa} / \mathrm{s}$ sample rate and 33 $\mathrm{GHz}$ analog bandwidth. The digitalized signal is equalized and recovered by off-line DSP algorithms. Then the LDPC decoder performs the sum-product algorithm with a maximum of 50 iterations.

\subsection{Results and analysis}

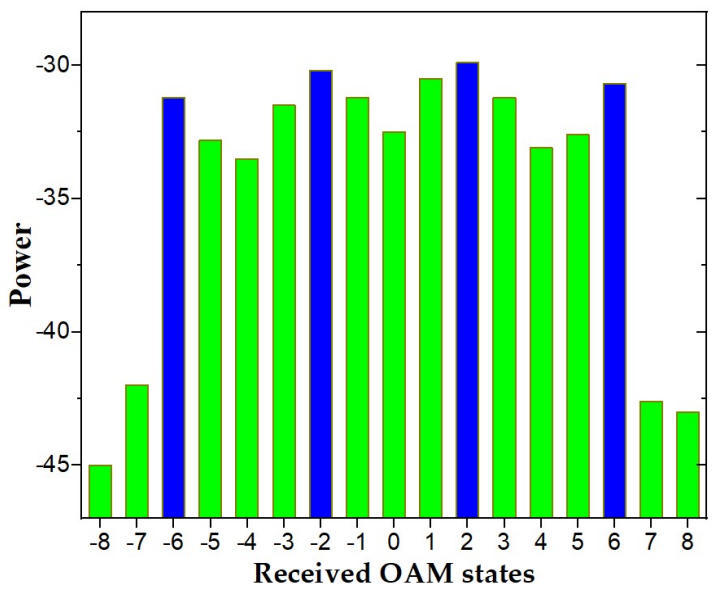

(a)

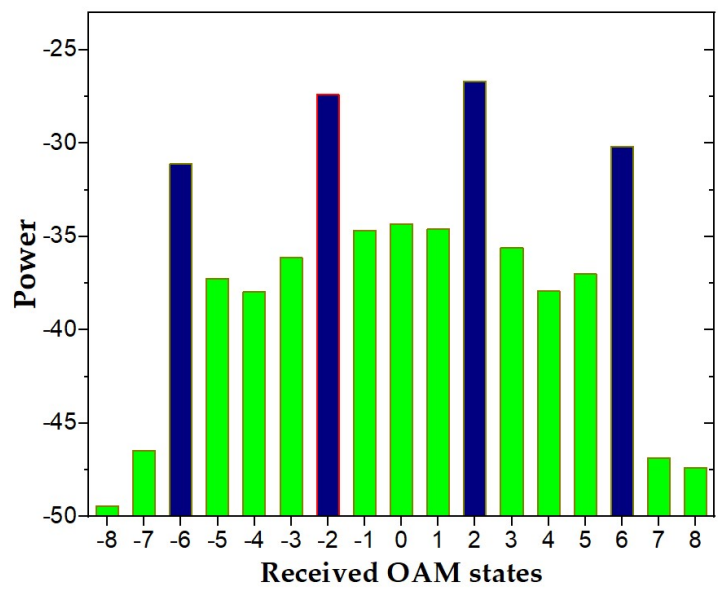

(b)

Figure 1. Measured power distributions of OAM channels: (a) without AO compensation and (b) with AO compensation.

This section may be divided by subheadings. It should provide a concise and precise description of the experimental results, their interpretation as well as the experimental conclusions that can be drawn. We firstly investigate the turbulence effects and the AO compensation performance. The power distributions over the target OAM channels (OAM modes of state indices $\pm 2, \pm 6$ ), and the adjacent channels are measured for crosstalk evaluation. As shown in Figure 1 (a), the mode crosstalk among OAM channels without compensation becomes very serious. The power of desired OAM channels is comparable to the undesired OAM channels. After AO compensation, as shown in Figure 
1 (b), the average extinction ratio can be improved to $\sim 6 \mathrm{~dB}$. Then, the effects of turbulence on system performance and the merits of AO correction and LDPC code are investigated.

Figure (a) shows the measured average BER performance in the absence and presence of turbulence when both AO compensation and LDPC code are not used. We can see that BER curves without turbulence will drop slowly with the increase of OSNR. This scenario is mainly caused by the imperfect phase patterns loaded on the SLM screens and the imperfect flatten pattern loaded on the DM. The pattern performance is limited by finite resolution, and imperfect Gamma correction, et al. What's worse, the BER curves in the presence of strong turbulence barely drop even in the regime of high OSNR because of the effect of severe mode crosstalk. Note that the degraded performance of OAM states of \pm 6 is not only due to the mode crosstalk, but also due to the boundary effect. The error floor of BER is beyond the error correction threshold of our LDPC code, which is of $\sim 0.04$. Therefore, AO correction is necessary to be used before LDPC coding to mitigate the mode crosstalk to certain acceptable level.

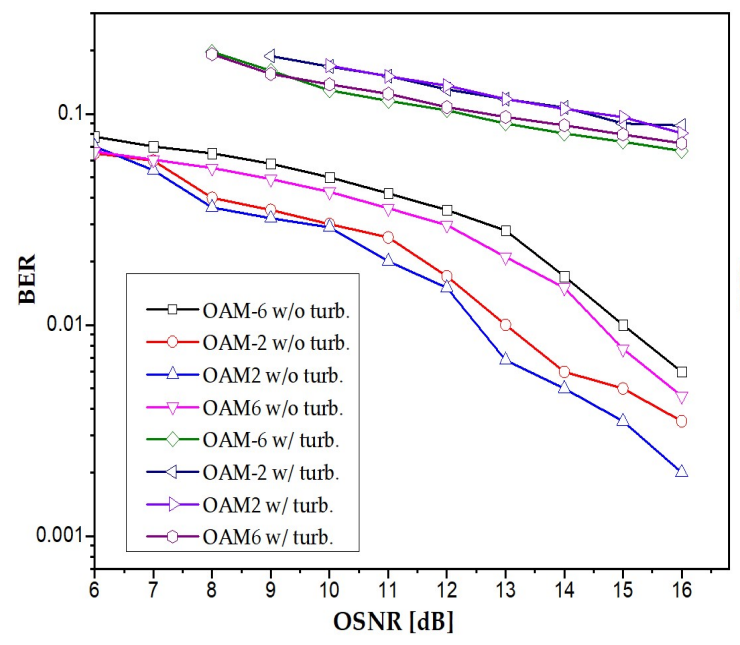

(a)

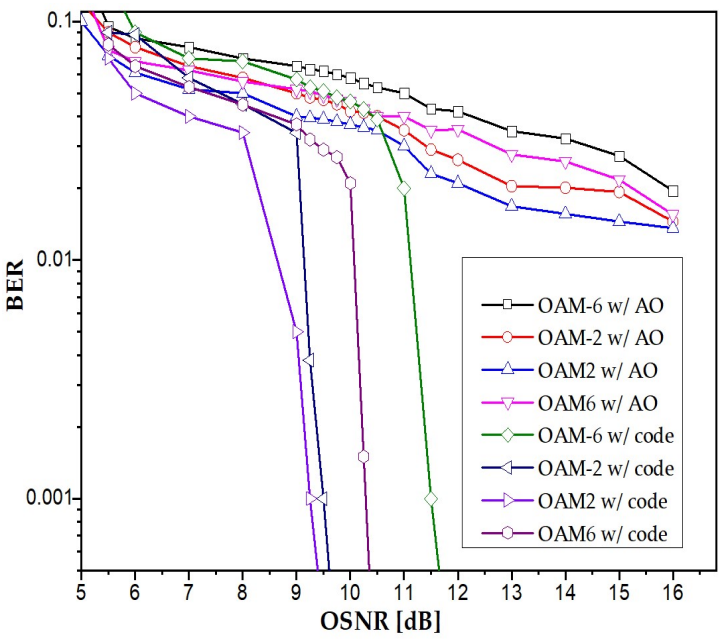

(b)

Figure 3. (a) BER versus received OSNR in the absence and presence of atmospheric turbulence. (b) BER vs. OSNR when AO correction and LDPC coding are used.

As shown in Figure (b), the system performance can be improved by combining AO correction and LDPC coding. When only AO correction is used, the BER curves drop slowly, but the BER values of all modes have been reduced below 0.04. Interestingly enough, the effects of our AO correction on OAM states of \pm 2 outperform that of OAM states of \pm 6 , which can be explained as follows. The first reason is due to the boundary effect, while the second can be contributed to the characteristics of the probe beam, which can more efficiently cover the small-size OAM beams. Once the AO correction is conducted, it is the time to use LDPC code to finally resolve the error floor. We can see from Fig. 5(b) that the BER curves will start to drop dramatically when OSNR is higher than $8 \mathrm{~dB}$. The coding gains of $3.9,4.1,5.2$, and $5 \mathrm{~dB}$ can be obtained respectively at $\mathrm{BER}=2 \times 10^{-2}$ for OAM states $2,-2,6$, and -6 .

\section{Joint Huffman and LDPC coding enabled free-space optical communication}

\subsection{Experimental setup}



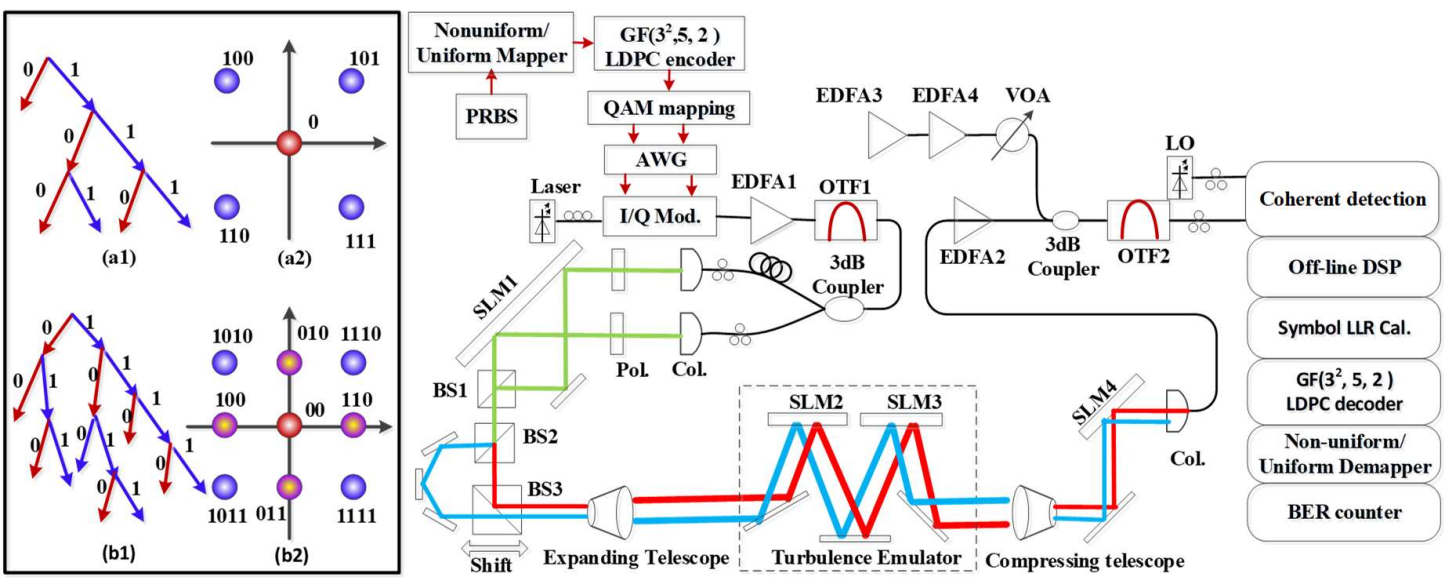

Figure 4. Experimental setup for Huffman coding enabled FSO communication systems. Insets: Huffman procedure (a1) and the corresponding constellation diagram (a2) for 5-QAM; Huffman procedure (b1) and the corresponding constellation diagram (b2) for 9-QAM.

Our experimental setup is depicted in Figure. At the transmitter side, the CW light with the wavelength centered at $1550 \mathrm{~nm}$ is sent to an I/Q modulator. The binary data sequences are nonuniformly mapped to symbols labeled by $0-4$ for 5-QAM, and 0-8 for 9-QAM by Huffman coding [26,30]; or uniformly mapped to symbols labeled by 0-3 for QPSK, and 0-7 for 8-QAM. Then the GF(5), $\mathrm{GF}\left(3^{2}\right)$ based LDPC encoding are applied to 5-QAM and 9-QAM sequences, respectively; while conventional GF(2) based LDPC encoding to QPSK (or 4-QAM) and 8-QAM. The Huffman procedures and the 5-QAM/9-QAM constellation diagrams are provided as insets Figure (a1-a2) and (b1-b2), respectively. Such obtained symbol sequences are pulse shaped with the help of an AWG to generate $12.5 \mathrm{G}$ Baud 4/5/8/9-QAM signals, and then used as the inputs of the I/Q modulator. The signals are amplified by an EDFA, and filtered with an OTF. The filtered signal is split by a 3-dB optical coupler, followed by an optical delay line applied in one path to decorrelate the data sequences. Each beam is collimated and converted to OAM beams by a reflective phase-only SLM. The left and right halves of the SLM1 screen are loaded with different phase patterns to convert the incoming Gaussian beams to OAM beams (OAM modes with state indices of 2 and -6). A blazed "fork" phase pattern is superimposed to separate the desirable OAM beams from the Gaussian beams. The generated OAM beams are then combined by a beam splitter-1 (BS1). The second BS (BS2) is used to split the multiplexed OAM beams into two branches, which will be combined by BS3. One branch is reflected once to form the OAM beams with inversed states (OAM states of -2 and 6); while the second one is reflected four times and delayed by $\sim 90$ symbols for data decorrelation. The spatial offset between the branches is realized by horizontally shifting the position of BS3. It is worthwhile to mention that each of the four OAM modes can be in principle spatially offset, but the space efficiency will be reduced accordingly [31]. In our FSO system, the rectangular aperture of the SLM screen restricts the available dimension for spatial offset to the horizontal axis. In order to maximally avoid the side-effect induced crosstalk, OAM states 2 and -6 are centrally aligned, but spatially offset against their inverses states in horizontal axis.

After passing through the telescope system, the offset beams are reflected twice on SLM screens, loaded with randomly generated azimuthal phase patterns following Andrews' spectrum [29]. The time-varying atmospheric turbulence is emulated by changing the phase patterns at $50 \mathrm{~Hz}$ rate. This turbulence model is built on the Rytov variance of $\sigma_{R}^{2}=0.5$. The beams at the output of the turbulence emulator are collected by a compressing telescope, and sent to the SLM4 loaded with a phase pattern to demultiplex and convert the target OAM beam back to Gaussian beam. The converted beam is coupled into the SMF and amplified by an EDFA. The ASE noise-loading stage is constructed by two EDFAs cascaded with a VOA. The noisy signal is filtered by an OTF, and detected by a coherent receiver, which includes an optical $90^{\circ}$ hybrid, two BPDs, and a real-time oscilloscope. The digitalized signal is recovered by off-line DSP algorithms. After determining the symbol LLRs, 
the binary/nonbinary LDPC decoder performs the sum-product algorithm with maximum of 40 iterations. Finally, the BERs are counted for performance analysis.

\subsection{Results and analysis}

We first investigate the gain of signal to crosstalk ratio (SCTR) by spatial offset, which is shown in Figure. The SCTR gain is calculated by the power of the target OAM mode divided by that of the adjacent modes when only the target OAM beam is transmitted, which is illustrated as insets Figure (a1-a2). Because of the symmetry between OAM modes with positive and negative states, only the performances of OAM states of 2 and -6 are analyzed in our experiment. We find that SCTR gain of $>1.6 \mathrm{~dB}$ can be obtained for OAM state of 2; and $1 \mathrm{~dB}$ SCTR gain is achievable for OAM state of -6 . The optimum spatial offset is about $6 \mathrm{~mm}$ and $5 \mathrm{~mm}$ for OAM states of 2, and -6 , respectively. In our following experiment, the spatial offset is set to $5 \mathrm{~mm}$. Notice that the optimum spatial offset is mainly determined by the turbulence strength and the apertures of the optical components used in the FSO link.
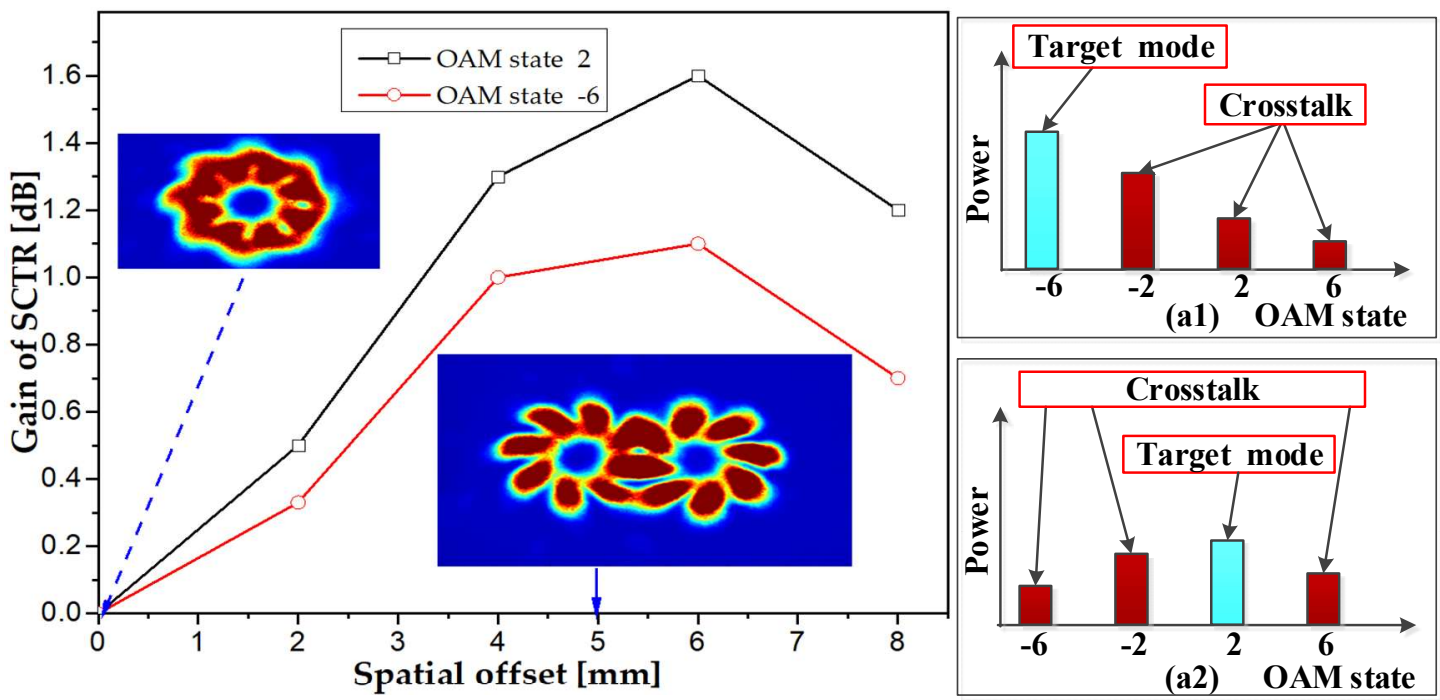

Figure 5. Gain of SCTR as a function of spatial offset. Insets: Illustrated SCTR calculation for (a1) OAM state of -6 , and (a2) OAM state of 2.

The performance of the LDPC-coded ununiform signaling will be evaluated next in the absence and presence of turbulence. Figure Error! No text of specified style in document. shows the measured BERs vs. OSNR performance in the absence of turbulence. We find from Figure Error! No text of specified style in document. (a-b) that the performance of uncoded 5-QAM is inferior to that of the uncoded QPSK because of the DSP penalty; the performance of uncoded 9-QAM is comparable to that of the uncoded 8-QAM. While the gaps between the coded QPSK and 5-QAM are less than 0.3 $\mathrm{dB}$, and $0.2 \mathrm{~dB}$ at $\mathrm{BER}=10^{-4}$, when OAM states of 2 and -6 are detected, respectively; $\mathrm{GF}\left(3^{2}\right)$ based 9QAM can outperform GF(2) based 8-QAM by $2.7 \mathrm{~dB}$, and $3.2 \mathrm{~dB}$ at BER=10-4, when OAM states of 2 and -6 are detected, respectively. Notice that the crosstalk among OAM modes still exists due to the imperfect phase patterns loaded onto the SLM screens. 


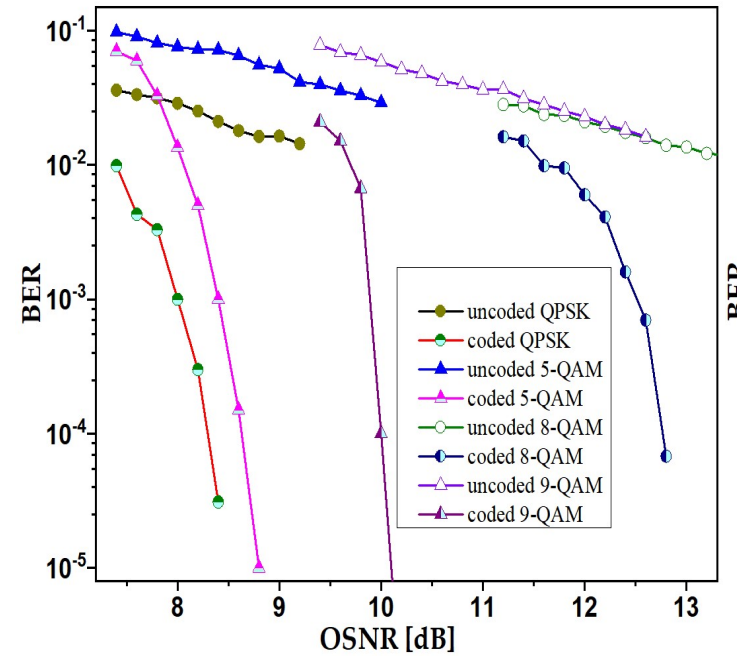

(a)

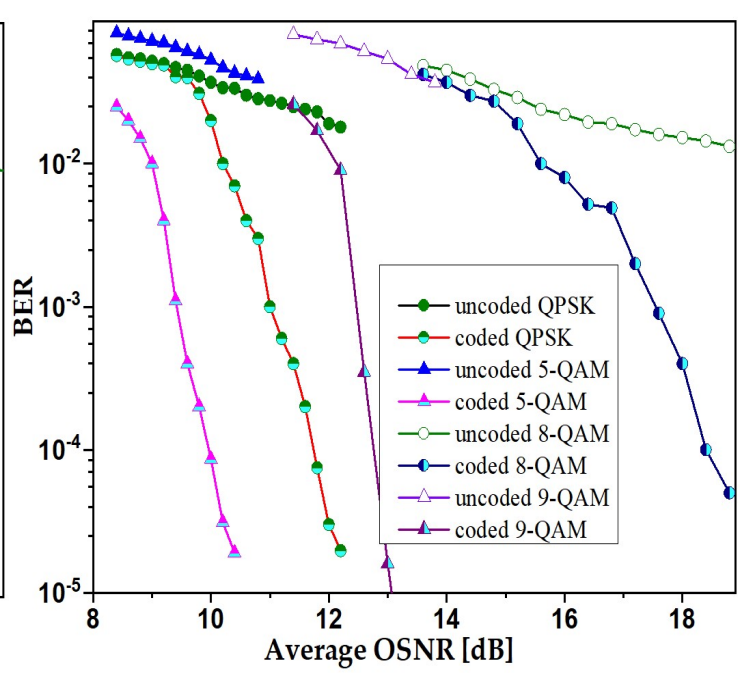

(b)

Figure Error! No text of specified style in document.. BER versus received OSNR in the absence of atmospheric turbulence, in cases that (a) OAM state 2 is detected, (b) OAM state -6 is detected.

The measured BERs vs. average OSNR performance in the presence of turbulence, are depicted in Figure. Each point is measured with 100 times' turbulence realizations. Compared to the uncoded curves shown in Figure Error! No text of specified style in document. (a-b), the gaps between uncoded QPSK and 5-QAM are closer in the presence of turbulence; the performance of uncoded 9-QAM is superior to that the uncoded 8-QAM. As shown in Figure (a), when OAM state 2 is detected, by comparing the coded QPSK and 8-QAM curves, the performance improvement of $>1.6 \mathrm{~dB}$ and $5.6 \mathrm{~dB}$ can be obtained via 5-QAM and 9-QAM at BER $=10^{-4}$, respectively. In case that OAM state of -6 is detected, the coding gains, as described in Figure (b), can be achieved of $>1.1 \mathrm{~dB}$ and $5.4 \mathrm{~dB}$ at $\mathrm{BER}=10^{-4}$ by comparing 5-QAM to QPSK, and 9-QAM to 8-QAM, respectively. Notice that the performance gains achieved by 5-QAM and 9-QAM are contributed by the OSNR gain, as well as the better turbulence tolerance.

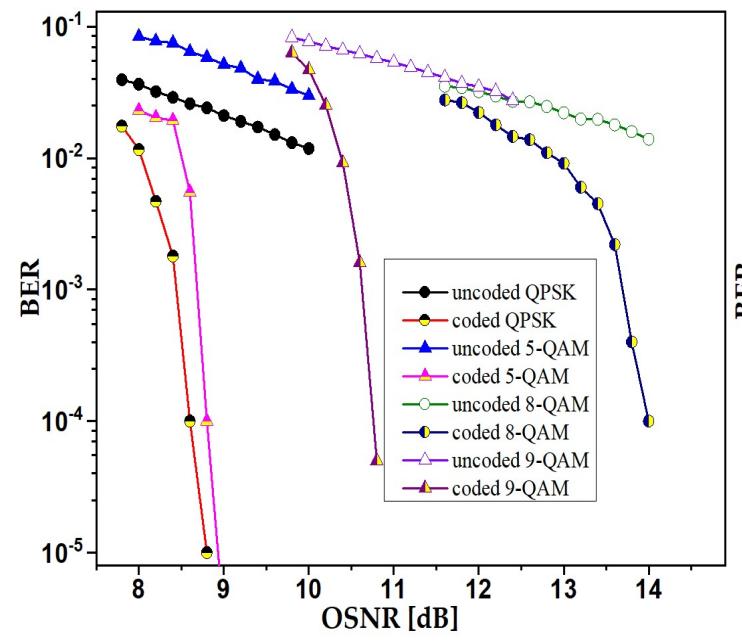

(a)

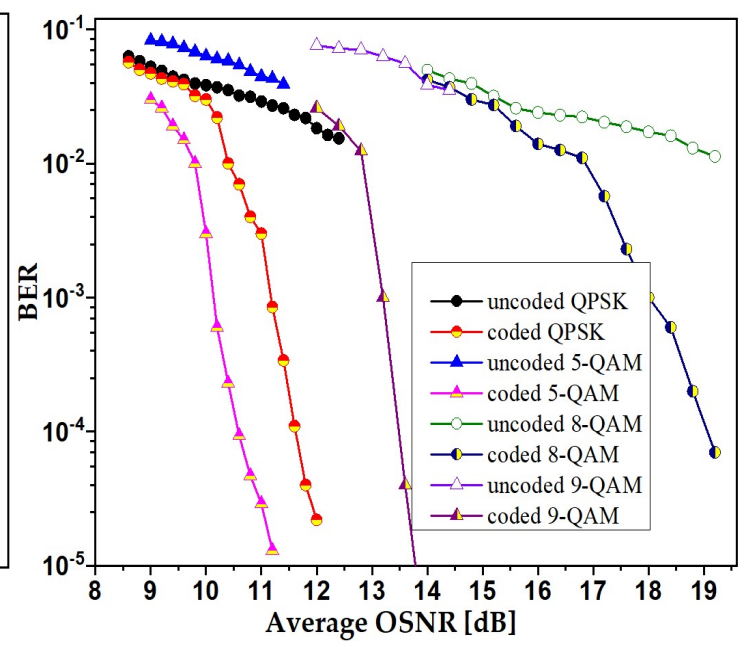

(b)

Figure 7. BER versus average OSNR in the presence of atmospheric turbulence, in cases that (a) OAM state 2 is detected, (b) OAM state -6 is detected.

For a better understanding of the benefits obtained by using nonuniform signaling, the minimum OSNR requirements at $\mathrm{BER}=10^{-4}$ for QPSK, 5-QAM, 8-QAM, and 9-QAM are listed in Table 1 , when OAM states 2 and 6 are observed, in the presence and absence of atmospheric turbulence. 
The turbulence induced penalties for OAM state 2, can be obtained as $3.3 \mathrm{~dB}, 1.4 \mathrm{~dB}, 5.7 \mathrm{~dB}$, and 2.8 $\mathrm{dB}$ for QPSK, 5-QAM, 8-QAM and 9-QAM, respectively. Meanwhile, the crosstalk penalty for OAM state -6 , can be obtained as $3.1 \mathrm{~dB}, 1.8 \mathrm{~dB}, 5 \mathrm{~dB}$, and $2.8 \mathrm{~dB}$ for QPSK, 5-QAM, 8-QAM, and 9-QAM, respectively. It can be concluded that the proposed 5-QAM and 9-QAM have a better crosstalk tolerance than regular QPSK and 8-QAM. It is because zero-power symbols are transmitted more frequently in 5-QAM and 9-QAM symbol sequences. When mode crosstalk occurs, these zero-power symbols will not interfere with the symbols carried among adjacent channels.

Table 1. Minimum OSNR requirements at BER of $10^{-4}$.

\begin{tabular}{|c|c|c|c|c|c|}
\hline & $\begin{array}{l}\text { Modulation } \\
\text { formats }\end{array}$ & QPSK & 5-QAM & 8-QAM & 9-QAM \\
\hline \multirow{2}{*}{ 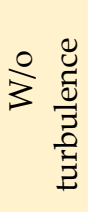 } & OAM state 2 & $8.3 \mathrm{~dB}$ & $8.6 \mathrm{~dB}$ & $12.7 \mathrm{~dB}$ & $10 \mathrm{~dB}$ \\
\hline & OAM state 6 & $8.6 \mathrm{~dB}$ & $8.8 \mathrm{~dB}$ & $14.0 \mathrm{~dB}$ & $10.8 \mathrm{~dB}$ \\
\hline \multirow{2}{*}{ 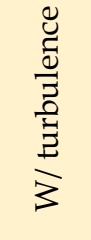 } & OAM state 2 & $11.6 \mathrm{~dB}$ & $10 \mathrm{~dB}$ & $18.4 \mathrm{~dB}$ & $12.8 \mathrm{~dB}$ \\
\hline & OAM state 6 & $11.7 \mathrm{~dB}$ & $10.6 \mathrm{~dB}$ & $19.0 \mathrm{~dB}$ & $13.6 \mathrm{~dB}$ \\
\hline
\end{tabular}

\section{Concluding remarks}

We have experimentally studied OAM-based FSO transmission system using LDPC coded QPSK modulation format. Four OAM modes each carrying 15.6 GBaud QPSK signals have been transmitted among the five WDM channels to realize $500 \mathrm{~Gb} / \mathrm{s}$ FSO transmission system per single polarization state. The mode crosstalk has been first mitigated by the wavefront sensorless adaptive optics system, and the residual crosstalk has been resolved by using sufficiently strong LDPC code. The coding gains of $3.9,4.1,5.2$, and $5 \mathrm{~dB}$ have been obtained at $\mathrm{BER}=2 \times 10^{-2}$ for OAM states $2,-2$, 6 , and -6 , respectively. By using frequency-locked lasers, beyond $1 \mathrm{~Tb} / \mathrm{s}$ serial optical transmission over strong atmospheric turbulence channel would be possible.

We also presented a two-stage crosstalk mitigation method in an OAM-based FSO communication system by combining spatial offset and LDPC-coded nonuniform signaling. The SCTR gain of $>1.6 \mathrm{~dB}$ and $1 \mathrm{~dB}$ were obtained for OAM states of 2, and -6 , respectively. The performances of 5-QAM and 9-QAM, based on joint Huffman coding and nonbinary LDPC codes, could outperform QPSK and 8-QAM by $>1.6 \mathrm{~dB}$ and $5.6 \mathrm{~dB}$ at $\mathrm{BER}=10^{-4}$, respectively, for OAM state of 2; the coding gains for OAM state of -6 , were achieved $>1.1 \mathrm{~dB}$ and $5.4 \mathrm{~dB}$ at $\mathrm{BER}=10^{-4}$ when comparing 5-QAM to QPSK, and 9-QAM to 8-QAM, respectively.

Author Contributions: This research study was supervised by Dr. Dordjevic. All laboratory experimentations, results, and analysis were done by Zhen Qu.

Funding: This research was funded by ONR grant number [N00014-13-1-0627].

Conflicts of Interest: The authors declare no conflict of interest.

\section{References}


1 Qu, Z.; Fu, S.; Zhang, M.; Tang, M.; Shum, P.; Liu, D. Analytical investigation on self-homodyne coherent system based on few-mode fiber. IEEE Photon. Technol. Lett. 2014, 26, 74-77.

2 Qu Z.; Li, Y.; Mo, W.; Yang, M.; Zhu, S.; Kilper D.; Djordjevic, I.B. Performance optimization of PM-16QAM transmission system enabled by real-time self-adaptive coding. Optics Lett. 2017, 42, 4211-4214.

3 Ip, E.; Lau, A. P. T.; Barros, D. J. F.; Kahn, J. M. Coherent detection in optical fiber systems. Opt. Express 2008, 16, 753-791.

4 Li, Y.; Yang, M.; Mo, W.; Zhu, S.; Qu, Z.; Djordjevic, I.B.; Kilper, D. Hysteresis-based Margin Allocation for Adaptive Coding in SDN-enabled Optical Networks. In Proceedings of the Optical Fiber Communication Conference (OFC), San Diego, California, USA. 11-15 March 2018, pp. Th1D-2.

5 Qu, Z.; Djordjevic, I. B. Experimental evaluation of LDPC-coded OAM based FSO communication in the presence of atmospheric turbulence. In Proceedings of the 12th International Conference on Telecommunications in Modern Satellite, Cable and Broadcasting Services (TELSIKS), Nis, Serbia, 14- 17 October 2015; pp. 117-122.

6 Djordjevic, I. B; Qu, Z. Coded Orbital Angular Momentum Modulation and Multiplexing Enabling UltraHigh-Speed Free-Space Optical Transmission; Uysal M., Capsoni C., Ghassemlooy Z., Boucouvalas A., Udvary E., Optical Wireless Communications Signals and Communication Technology, Springer, Cham: 2016; pp. 363-385, ISBN 978-3-319-30200-3.

7 Yue, Y.; Huang, H.; Ahmed, N.; Yan, Y.; Ren, Y.; Xie, G.; Rogawski, D.; Tur, M.; Willner, A. E. Reconfigurable switching of orbital-angular-momentum-based free-space data channels. Opt. Lett. 2013, 38, 5118-5121.

8 Xie, Y.; Geng, Z.; Kong, D.; Zhuang, L.; Lowery, A. J. Selectable-FSR 10-GHz granularity WDM superchannel filter in a reconfigurable photonic integrated circuit. J. Lightwave Technol. 2018, 36, 2619-2626.

9 Xie, Y.; Geng, Z.; Zhuang, L.; Burla, M.; Taddei, C.; Hoekman, M.; Leinse, A.; Roeloffzen, C.G.; Boller, K.J.; Lowery, A.J. Programmable optical processor chips: toward photonic RF filters with DSP-level flexibility and MHz-band selectivity. Nanophotonics 2017, 7, 421-454.

10 Lowery, A.J.; Xie, Y.; Zhu, C.; Systems performance comparison of three all-optical generation schemes for quasi-Nyquist WDM. Opt. Express 2015, 23, 21706-21718.

11 Qu, Z.; Zhang, S.; Djordjevic, I.B. Universal Hybrid Probabilistic-geometric Shaping Based on Twodimensional Distribution Matchers. In Proceedings of the Optical Fiber Communication Conference (OFC), San Diego, California, USA. 11-15 March 2018, pp. M4E.4.

12 Zhang, S.; Qu, Z.; Yaman, F.; Mateo, E.; Inoue, T.; Nakamura, K.; Inada, Y.; Djordjevic, I.B. Flex-Rate Transmission using Hybrid Probabilistic and Geometric Shaped 32QAM. In Proceedings of the Optical Fiber Communication Conference (OFC), San Diego, California, USA. 11-15 March 2018, pp. M1G.3.

13 Lin, C.; Qu, Z.; Liu, T.; Zou, D.; Djordjevic, I. B. Experimental study of capacity approaching general LDPC coded non-uniform shaping modulation format. In Proceedings of the Asia Communications and Photonics Conference (ACP), Wuhan, China, 2-5 November 2016, pp. AF3A.1.

14 Wang, J.; Yang, J.Y.; Fazal, I.M.; Ahmed, N.; Yan, Y.; Huang, H.; Ren, Y.; Yue, Y.; Dolinar, S.; Tur, M.; Willner, A.E. Terabit free-space data transmission employing orbital angular momentum multiplexing. Nature Photonics 2012, 6, pp.488-496.

15 Djordjevic, I.B.; Qu, Z. Coded orbital-angular-momentum-based free-space optical transmission; Wiley Encyclopedia of Electrical and Electronics Engineering: Hoboken, NJ, USA, 2016.

16 Willner, A.E.; Huang, H.; Yan, Y.; Ren, Y.; Ahmed, N.; Xie, G.; Bao, C.; Li, L.; Cao, Y.; Zhao, Z.; Wang, J. Optical communications using orbital angular momentum beams. Advances in Optics and Photonics 2015, 7, pp.66-106. 
17 Allen, L.; Beijersbergen, M.W.; Spreeuw, R.J.C.; Woerdman, J.P. Orbital angular momentum of light and the transformation of Laguerre-Gaussian laser modes. Physical Review A 1992, 45, pp.8185.

$18 \mathrm{Qu}$, Z.; Djordjevic, I.B. LDPC-coded OAM based FSO transmission system in the presence of strong atmospheric turbulence. In Proceedings of the Signals, Systems and Computers, 49th Asilomar Conference, Pacific Grove, California, USA, 8-11 November 2015, pp. 999-1002.

$19 \mathrm{Qu}, \mathrm{Z}$; Djordjevic, I.B. Beyond $1 \mathrm{~Tb} / \mathrm{s}$ free-space optical transmission in the presence of atmospheric turbulence. In Proceedings of the Photonics North (PN), Ottawa, Ontario, Canada, 6-8 June 2017, pp. 1-1.

20 Ren, Y.; Xie, G.; Huang, H.; Li, L.; Ahmed, N.; Yan, Y.; Lavery, M.P.; Bock, R.; Tur, M.; Neifeld, M.A.; Boyd, R.W.Turbulence compensation of an orbital angular momentum and polarization-multiplexed link using a data-carrying beacon on a separate wavelength. Optics Lett. 2015, 40, pp.2249-2252.

$21 \mathrm{Qu}, \mathrm{Z}$. and Djordjevic, I.B. $500 \mathrm{~Gb} / \mathrm{s}$ free-space optical transmission over strong atmospheric turbulence channels. Optics Lett. 2016, 41, pp.3285-3288.

22 Huang, H.; Cao, Y.; Xie, G.; Ren, Y.; Yan, Y.; Bao, C.; Ahmed, N.; Neifeld, M.A.; Dolinar, S.J.; Willner, A.E. Crosstalk mitigation in a free-space orbital angular momentum multiplexed communication link using $4 \times 4$ MIMO equalization. Optics Lett. 2014, 39, pp.4360-4363.

23 Ren, Y.; Wang, Z.; Xie, G.; Li, L.; Cao, Y.; Liu, C.; Liao, P.; Yan, Y.; Ahmed, N.; Zhao, Z.; Willner, A.E. Freespace optical communications using orbital-angular-momentum multiplexing combined with MIMO-based spatial multiplexing. Optics let. 2015, 40, pp.4210-4213.

$24 \mathrm{Qu}, \mathrm{Z}$.; Djordjevic, I.B. Coded orbital angular momentum based free-space optical transmission in the presence of atmospheric turbulence. In Proceedings of the Asia Communications and Photonics Conference (ACP), Hong Kong, 19-23 November 2015, pp. AS3D-3.

$25 \mathrm{Qu}, \mathrm{Z}$; Djordjevic, I.B. Approaching terabit optical transmission over strong atmospheric turbulence channels. In Proceedings of the 18th International Conference on Transparent Optical Networks (ICTON), Trento, Italy, 10-14 July 2016, pp. 1-5.

26 Liu, T.; Qu, Z.; Lin, C.; Djordjevic, I.B. Nonuniform signaling based LDPC-coded modulation for high-speed optical transport networks. In Proceedings of the Asia Communications and Photonics Conference (ACP), Wuhan, China, 2-5 November 2016, paper AF3D.5.

$27 \mathrm{Qu}, \mathrm{Z}$;; Djordjevic, I.B. FEC Coding for Nonuniform QAM. In Proceedings of the Signal Processing in Photonic Communications (SPPCom), New Orleans, Louisiana, USA, 24-27 July 2017, paper SpTu3E.2.

28 Qu, Z.; Lin, C.; Liu, T.; Djordjevic, I.B. Experimental study of nonlinearity tolerant modulation formats based on LDPC coded non-uniform signaling. In the Optical Fiber Communication Conference(OFC), Los Angeles, California, USA,19-23 March 2017, pp. W1G-7.

29 Andrews, L.C.; Phillips, R.L.; Hopen, C.Y., 2001. Laser beam scintillation with applications; SPIE press: 2001, ISBN 9781510604896

30 Qu, Z.; Lin, C.; Liu, T.; Djordjevic, I.B. Experimental Investigation of GF (3 (exp 2)) Nonbinary LDPC-coded Non-uniform 9-QAM Modulation Format. In Proceedings of the 42nd European Conference on Optical Communication (ECOC), Dusseldorf, Germany, 18-22 September 2016, pp.1-3 